LA ENSEÑANZA DE LA METODOLOGÍA 



\title{
Diseño y desafíos metodológicos de la investigación feminista en ciencias sociales
}

\author{
Design and methodological challenges of feminist research in \\ social sciences
}

\author{
ROCÍO JiMÉNEZ CORTÉS \\ Universidad de Sevilla \\ rjimenez@us.es (ESPAÑA)
}

Recibido: 25.042019
Aceptado: 15.02 .2021

\section{RESUMEN}

La investigación feminista genera otras formas de hacer ciencia. En el ámbito de las ciencias sociales, aún sigue resultando desconocida y controvertida desde el punto de vista metodológico. Las epistemologías feministas, sus principios y valores marcan las directrices metodológicas de este tipo de investigación. Hay escasos trabajos que se centren en aportar pautas aplicadas y sintéticas de lo que implica una buena práctica de investigación desde este enfoque. Por ello, nos proponemos generar, desde la evidencia, una guía con pasos metodológicos que permitan planear y diseñar la investigación feminista. También, perseguimos identificar en la literatura científica los principales desafíos metodológicos a los que se enfrenta en la actualidad este tipo de investigación. Así, realizamos una revisión de literatura reciente y referente en la materia, seleccionando tanto estudios empíricos y que recogen casos ilustrativos de investigaciones feministas como reflexiones metodológicas y revisiones publicadas en revistas de una diversidad de áreas de ciencias sociales y que cuentan con revisión por pares. El análisis de las contribuciones se realiza desde un enfoque temático y de teoría fundamentada, siguiendo procesos de codificación abierta y abductiva, donde se tienen en cuenta conceptos nucleares para las epistemologías feministas. Los procesos de análisis buscan la proyección aplicada de principios y valores de las epistemologías feministas en una estructura interpretativa que guíe y oriente la investigación. El aporte final supone disponer de una guía fundamentada y ejemplificada que permite articular futuros diseños de investigación, alertando sobre aspectos metodológicos clave para tener en cuenta. Así como también, se identifican cuatro desafíos metodológicos principales que mantienen el debate abierto en la academia en torno a la investigación feminista actual. En última instancia, 
el estudio invita a la reflexión sobre la tensión entre el ideal de investigación feminista, la práctica actual de esta investigación y las posibilidades metodológicas que implica su puesta en marcha.

\title{
PALABRAS CLAVE
}

Investigación feminista; investigación social; metodología; conocimiento científico; guía de investigación.

\begin{abstract}
Feminist research generates other ways of doing science. In the field of social sciences, it is still unknown and controversial from a methodological point of view. Feminist epistemologies, their principles and values set the methodological guidelines for this type of research. There are few works that focus on providing applied and synthetic guidelines of what a good research practice implies from this approach. Therefore, we propose to generate, from the evidence, a guide with methodological steps that allow planning and designing feminist research. Also, we seek to identify in the scientific literature the main methodological challenges that this type of research currently faces. Thus, we conducted a review of recent and benchmark literature on the subject, selecting both empirical studies that collect illustrative cases of feminist research as well as methodological reflections and reviews published in journals from a variety of areas of social sciences and that have peer review. The analysis of the contributions is carried out from a thematic and grounded theory approach, following open and abductive coding processes, where core concepts for feminist epistemologies are taken into account. The analysis processes seek the applied projection of principles and values of feminist epistemologies in an interpretive structure that guides and orients the investigation. The final contribution involves having a well-founded and exemplified guide that allows the articulation of future research designs, warning about key methodological aspects to take into account. As well as, four main methodological challenges are identified that keep the debate open in academia around current feminist research. Ultimately, the study invites reflection on the tension between the ideal of feminist research, the current practice of this research and the methodological possibilities that its implementation implies.
\end{abstract}

\section{KEY WORDS}

Feminist research; social research; methodology; scientific knowledge; research guides. 


\section{INTRODUCCIÓN}

Encontramos escasos aportes científicos que traduzcan los principios feministas en una buena práctica de investigación. Y, además, ofrezcan a quien se interesa por la investigación feminista una serie de pautas metodológicas concretas y aplicadas para producir conocimiento científico (Leavy y Harris, 2019). En este sentido, la relevancia y originalidad del trabajo que presentamos ahonda en estos aspectos.

La investigación feminista a pesar de que cuenta con una trayectoria amplia en diferentes áreas de conocimiento y ciencias sociales sigue resultando desconocida y controvertida desde el punto de vista metodológico (Bishop, 2018; Kaur y Nagaich, 2019). Según Ruan (2020), la investigación feminista se define por sus preguntas de investigación (si se trata principalmente de vida y experiencia de las mujeres), por la lente teórica del proyecto, y por el uso de metodología de investigación feminista. Por eso, consideramos clave en el momento actual que la investigación feminista cuente con pautas didácticas que, de forma sintética y muy ilustrada, faciliten la enseñanza de sus métodos y formen en las implicaciones de sus epistemologías y procesos. En este sentido, el trabajo que presentamos hace este aporte.

Los objetivos que perseguimos son: 1) generar, desde la evidencia científica, una guía con pautas metodológicas para el diseño de la investigación feminista que sirva como material didáctico para su uso por estudiantes que se inician en la carrera científica desde diferentes Ciencias Sociales y por aquellas personas que se interesan en profundizar en las diferencias metodológicas que dotan de identidad a este tipo de investigación y 2) identificar en la literatura científica los principales desafíos metodológicos a los que se enfrenta en la actualidad la investigación feminista derivados de las epistemologías y principios asumidos por la teoría feminista.

Para dar cobertura a estos objetivos se realiza una revisión de la literatura científica priorizando aportes de los últimos cinco años (2016-2020) a través de Google Académico partiendo como criterio de la selección de trabajos (tanto teóricos como empíricos) que contribuyan a las discusiones metodológicas en el campo de las ciencias sociales. Por ello, se tienen en cuenta tanto reflexiones metodológicas y revisiones, como estudios empíricos o contribuciones que describen casos ilustrativos en el ámbito de la investigación feminista y que están publicados en revistas que cuentan con revisión por pares. Para la búsqueda se emplea como descriptor "feminist research methodology". La siguiente tabla muestra una selección de 20 trabajos actuales que junto con otra literatura científica dan cobertura a los objetivos perseguidos. La tabla 1 recoge también la revista científica mostrando el área de conocimiento dentro de las ciencias sociales del que proceden los trabajos. 
Tabla 1. Selección de literatura científica de los últimos cinco años

\begin{tabular}{|c|c|c|}
\hline $\begin{array}{l}\text { Tipo de } \\
\text { aporte }\end{array}$ & Autoría y año & Revista científica \\
\hline \multirow{8}{*}{\begin{tabular}{|l}
0 \\
0 \\
0 \\
0 \\
0 \\
0 \\
0 \\
0 \\
0 \\
0 \\
0 \\
0 \\
0 \\
0 \\
0 \\
0 \\
0 \\
0 \\
0 \\
0 \\
0 \\
0 \\
0 \\
0 \\
0 \\
0 \\
0 \\
0 \\
0 \\
0 \\
0
\end{tabular}} & $\begin{array}{l}\text { Cuklanz, L., y Rodríguez, M. P. } \\
(2020) .\end{array}$ & Investigaciones Feministas \\
\hline & Walters, R. (2020) & Qualitative Research \\
\hline & Åhäll, L. (2018) & International Political Sociology \\
\hline & Fraser, H., y MacDougall, C. (2017). & Qualitative Social Work \\
\hline & $\begin{array}{l}\text { Pérez, L. T., Valderrama, C. G., y } \\
\text { Álvarez, C. (2017) }\end{array}$ & Psicoperspectivas \\
\hline & Attia, M., y Edge, J. (2017) & $\begin{array}{l}\text { Open Review of Educational Re- } \\
\text { search }\end{array}$ \\
\hline & Díaz, A. A., y García, R. G. (2017) & $\begin{array}{l}\text { EMPIRIA. Revista de Metodología } \\
\text { de las Ciencias Sociales }\end{array}$ \\
\hline & $\begin{array}{l}\text { Van Der Haar, M., y Verloo, M. } \\
(2016)\end{array}$ & Politics \& Gender \\
\hline \multirow{12}{*}{ 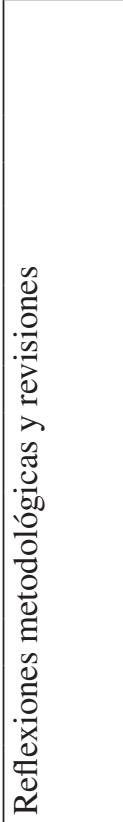 } & $\begin{array}{l}\text { Astudillo-Mendoza, P. A., Figueroa- } \\
\text { Quiroz, V. A., y Cifuentes-Zunino, } \\
\text { F. (2020) }\end{array}$ & Investigaciones Feministas \\
\hline & Ruan, N. (2020) & Qualitative Research \\
\hline & Gordon, R. (2019) & Gender \& Development \\
\hline & $\begin{array}{l}\text { Wigginton, B., y Lafrance, M. N. } \\
(2019)\end{array}$ & Feminism \& Psychology \\
\hline & $\begin{array}{l}\text { Cooky, C., Linabary, J. R., y Corple, } \\
\text { D. J. (2018) }\end{array}$ & Big Data \& Society \\
\hline & Bishop, S. (2018) & Feminist Media Studies \\
\hline & Biglia, B., y Bonet i Martí, J. (2017) & $\begin{array}{l}\text { Annual Review of Critical Psycho- } \\
\text { logy }\end{array}$ \\
\hline & $\begin{array}{l}\text { Linabary, J. R., y Hamel, S. A. } \\
(2017)\end{array}$ & Feminist Review \\
\hline & Rayaprol, A. (2016) & Contributions to Indian Sociology \\
\hline & Falconer, E. (2017) & Women's Studies International Forum \\
\hline & $\begin{array}{l}\text { Else-Quest, N. M. y Hyde J., S. } \\
\text { (2016a) }\end{array}$ & Psychology of Women Quarterly \\
\hline & $\begin{array}{l}\text { Else-Quest, N. M. y Hyde J., S. } \\
(2016 b)\end{array}$ & Psychology of Women Quarterly \\
\hline
\end{tabular}

Los artículos seleccionados se analizan en fases sucesivas desde un enfoque temático y de teoría fundamentada, siendo considerados en su globalidad como unidades de análisis. Se emplea un proceso de codificación abierta y abductiva, partiendo tanto de constructos claves implicados en las epistemologías feminis- 
tas (como son encarnar, objetividad fuerte, conocimiento situado, interseccionalidad,...) como de conceptos incluidos en los propios artículos relacionados con el diseño de la investigación feminista y las metodologías. La codificación abierta va dando paso a estructuras más complejas y relaciones entre constructos que nos permiten emplear redes semánticas que aportan estructuras interpretativas sobre el contenido de los artículos. Una síntesis de las principales categorías consideradas y sus relaciones se muestra en la Figura 1 a modo de esquema organizativo de pasos para el diseño de la investigación.

Tomando como base la revisión de literatura científica y la reflexión sobre la propia experiencia investigadora recogemos, en los siguientes apartados los resultados articulados en una narrativa que da forma a la guía obtenida para el diseño de la investigación feminista y a la identificación de los principales desafíos metodológicos a los que se enfrenta en la actualidad.

\section{GUÍA PARA EL DISEÑO DE LA INVESTIGACIÓN FEMINISTA}

La investigación feminista aborda el género como sujeto, por tanto, la vivencia del género y más allá del género (etnia, clase...) es el núcleo constitutivo de los procesos de construcción de conocimiento científico. El género no es visto como una categoría analítica sino como una categoría constitutiva de la práctica de investigación. De ahí que, la investigación feminista haga investigación con el género no sobre el género. Para Biglia y Bonet i Martí (2017: 1) la metodología feminista como práctica política, procesual y discursiva se construye desde "dentro" pero también "contra" la "normalidad" académica.

Somos conscientes de que la investigación feminista puede ser problemática porque no obedece a reglas concretas que permitan calificarla de "correcta" (Falconer, 2017). No obstante, asumimos el reto de esbozar cuáles serían los principales elementos en el diseño de una investigación feminista derivados de la revisión, interpretación y análisis de la literatura científica actual y de referencia.

Para diseñar la investigación feminista hay que tener en cuenta que se caracteriza por exteriorizar los procesos de investigación que en otras investigaciones quedan invisibilizados u ocultos (Biglia y Vergés, 2016). A continuación, mostramos la estructura interpretativa derivada del análisis emprendido y concretada en un esquema con tópicos y categorías que caracterizan el diseño de una investigación feminista. Con esta guía de pautas que vamos a exponer damos cobertura al primer objetivo de este trabajo. 


\title{
Figura 1. Diseño de la investigación feminista (elaboración propia)
}

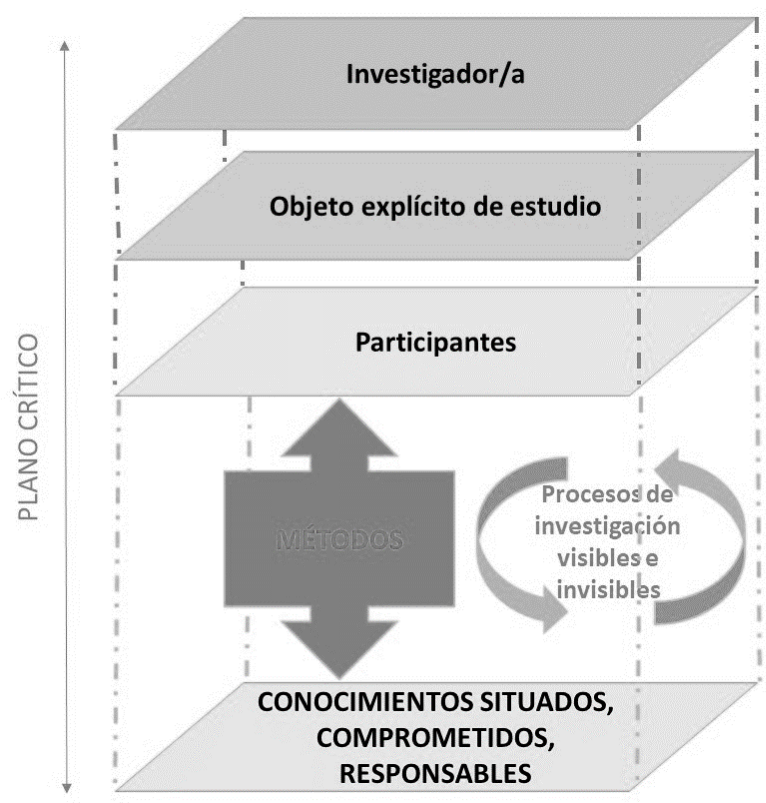

\author{
¿Quién investiga? \\ Postura epistemológica \\ Posición \\ Contexto \\ ¿El qué se investiga? \\ Tema, problema de investigación y \\ objetivos \\ Enfoque y marco teórico \\ ¿Con quién? \\ Participantes (género, edad, etnia...) \\ Posición \\ Contexto \\ ¿Cómo? \\ Métodos, técnicas y procesos \\ Ética \\ Limitaciones \\ ¿Para qué? \\ Resultados y consecuencias \\ Beneficiarios/as \\ Relevancia
}

\section{1. ¿Quién investiga?}

Las epistemologías feministas plantean formas muy concretas de producir conocimiento científico (Jiménez-Cortés, 2019). Estas perspectivas tienen que decidirse de forma consciente y hacerse explícitas cuando diseñamos la investigación. La epistemología feminista forma parte de la postura que adopta la investigadora o el investigador ante la misma. Conocer y dominar estas epistemologías es de vital importancia porque modelan las formas de producir conocimiento y, por tanto, el tipo de conocimiento que alcanzamos sobre lo estudiado. Así, expone Rayaprol (2016) cómo descartó plantear la investigación desde la epistemología del punto de vista feminista y decidió optar por una integración de enfoques de género:

"No privilegié las experiencias de las mujeres sobre las de los hombres y tampoco discutí sobre la perspectiva de cuál es la más precisa. Yo buscaba entender las experiencias particulares de las mujeres tal como las vemos dentro de sus propios contextos y empleé tanto los enfoques centrados en las mujeres como los integrados en el género" (2016: 380)

Además de hacer explícita la epistemología cuando se planea la investigación feminista resulta clave especificar el rol que asumimos. Nuestro rol como investigadores o investigadoras está en gran medida supeditado por estas perspectivas. Es decir, responder a las preguntas ¿cuáles son mis posicionamientos? 
¿con qué experiencias, creencias y formación me enfrento a la investigación? ¿en qué momento personal o profesional? también resultan claves. A menudo la persona que investiga no lo hace al margen de sus vivencias, su formación, sus creencias y sus circunstancias vitales. De ahí que, la investigación feminista esté sujeta a una autorreflexión personal de partida que haga explícito el posicionamiento de quien investiga. Resulta común encontrar verbalizados los posicionamientos de las investigadoras en este tipo de investigación y cuestionarlos conforme avanza el estudio a la luz de las epistemologías feministas, como mostramos en este ejemplo:

"Nos propusimos explicitar, tanto a las participantes como a quien pudiera interesarse en el estudio, nuestros posicionamientos como mujeres latinas/ chilenas, feministas, investigadoras sociales y usuarias de herramientas de comunicación online y nos comprometimos a desarrollar ejercicios reflexivos constantes respecto a nuestro quehacer a lo largo del proceso. Fruto de ello, surge el presente artículo, en el que compartimos algunas de las tantas reflexiones surgidas a raíz de las decisiones metodológicas que hemos tomado en la investigación, todas ellas revisadas a la luz de las epistemologías feministas y de algunas de sus propuestas respecto a cómo hacer ciencia feminista" (Astudillo-Mendoza, Figueroa-Quiroz, y Cifuentes-Zunino, 2020: 240)

Este ejercicio de posicionamiento es una manera de "encarnar" en los términos planteados por la epistemología feminista el proceso de investigación y contextualizar la experiencia. Como explican Araiza y González (2017) hay compromisos con las perspectivas teóricas que requieren revisión constante a nivel práctico cuando llevamos a cabo este tipo de investigación.

"Podemos decir que muchas reflexiones vienen de nuestro hacer, de nuestras prácticas encarnadas, las cuales nos llevaron a pensar en la teoría y a modificar nuestro ejercicio científico" (Araiza y González, 2017: 79).

\section{2. ¿Qué se investiga?}

La investigación feminista supone conocer los enfoques epistemológicos desde esta perspectiva, pero sobre todo tener sensibilidad hacia temas que tienen que ser investigados y que especialmente afectan a las mujeres y a sus experiencias particulares de vida. En la investigación feminista ser conscientes de quienes somos como personas y como investigadores o investigadoras resulta crucial incluso para el tema que abordamos. La selección del tema de investigación está muy vinculado a nuestra historia personal o profesional, a nuestros intereses, motivaciones y necesidades. En la investigación feminista, la propia trayectoria y contexto vital está en la base de la selección de los temas que investigamos. Priorizamos aquello que requiere atención y responde, a nuestro criterio, a aspiraciones de mejora. El siguiente fragmento extraído de la contribución de Attia y Edge (2017) ilustra lo comentado: 
"Cuestiones como la historia profesional, las relaciones colegiales y las aspiraciones de mejorar el uso de la tecnología en el programa de idiomas influyeron en mi elección del tema, la formulación de preguntas de investigación, la adopción del diseño de la investigación, la selección de métodos de recopilación de datos y el enfoque de representación y presentación de informes". (2017: en línea).

El tema de investigación da paso a preguntas específicas de investigación que pueden ser respondidas de forma operativa. Las preguntas informan sobre el sentido y la dirección del estudio en términos teóricos y metodológicos. Agee (2009) invita a pensar en la pregunta de investigación como un viaje. También la compara con la lente de una cámara fotográfica que tiene la posibilidad de enmarcar un paisaje y enfocar un conjunto específico de aspectos o acciones. Cuando comenzamos la investigación especialmente si tenemos poca experiencia a menudo el planteamiento de la pregunta o preguntas de investigación puede resultar complejo. A continuación, observamos la pregunta de investigación que se plantea Ruan (2020) sobre el liderazgo intelectual de las mujeres académicas en Hong Kong:

"Las mujeres académicas se encuentran en una situación relativamente desventajosa debido a los prejuicios y discriminaciones de género en las universidades y las comunidades académicas. Tienen que luchar y esforzarse para avanzar en su viaje académico buscando oportunidades y protegiendo sus espacios y libertad. Así que el estudio plantea la pregunta: ¿cómo ven y desarrollan las mujeres académicas el liderazgo intelectual?" (2020: 4)

La pregunta de investigación, aunque inicialmente es promovida por quien investiga en su empeño por conocer nuevos datos sobre una situación está condicionada por las personas que participan. Y es que las personas que participan pueden ayudar en la formulación de preguntas de investigación como implicadas y se plantean en un proceso interactivo. Por ello, siempre hay un avance inicial de la cuestión que se puede dejar abierta a revisiones, reelaboraciones y ajustes posteriores en contacto con participantes. En algunas ocasiones el contacto con ellas/os, ayuda a enfocarlas y definirlas, también el manejo de literatura especializada apoya el desarrollo de las preguntas de investigación.

Según Agee (2009) las preguntas de investigación requieren una reflexión sobre cómo afectarán a la vida de las personas participantes y cómo posicionan a la persona que investiga en relación con ellas. Especialmente cuando estudiamos aspectos de la vida de otras personas o estas forman parte de colectivos marginados o vulnerables. La revisión de los efectos que las preguntas de investigación pueden tener sobre personas y/o contextos debe ser una constante a lo largo del estudio.

En este sentido, también es importante que las preguntas de investigación se formulen de manera que repercutan en alguna forma de acción social, es decir, tengan una proyección práctica y aplicada. Es preciso valorar qué soluciones aporta. El siguiente ejemplo muestra cómo nace el tema de investigación, en qué preguntas se concreta y qué pretende aportar en última instancia: 
"En este contexto, en el que confluyen dos temas que nos apasionan, las tecnologías y los movimientos sociales de mujeres, nos desafiamos a desarrollar una investigación que fuera coherente con nuestro compromiso político feminista derivado de una voluntad específica de subvertir un orden androcéntrico que configura unas determinadas identidades sexuales normativas y genera efectos de dominación sobre las mujeres. Nos preguntamos entonces, por las relaciones de poder patriarcales que se ejercían en esta colectividad integrada exclusivamente por mujeres, por las regulaciones en las performances de género y también, si podíamos - o no- considerar este grupo, una comunidad" (Astudillo-Mendoza, Figueroa-Quiroz y Cifuentes-Zunino, 2020: 240).

\section{3. ¿CON QUIÉN/ES?}

La investigación feminista independientemente de las estrategias y técnicas de muestreo que siga para la incorporación de participantes a la investigación ha de ser sensible a sus experiencias. La experiencia del investigador o de la investigadora es un punto de atención que se sitúa en el mismo plano crítico que la experiencia y vidas de las o los participantes.

Un principio feminista clave cuando se trata de reflexionar con quién/es vamos a desarrollar la investigación es que hay que partir de la idea de que los/as participantes van a colaborar durante el proceso de investigación en un plano relacional simétrico. En este sentido, tenemos que reducir las diferencias de poder entre "nosotros/as" como investigadores/as y ellos/as como participantes. Para la investigación feminista (Nazneen, Darkwah y Sultan, 2014) la conciencia de la relación jerárquica entre investigadores y participantes, junto con los esfuerzos conscientes para igualar esta disparidad, es otro principio básico de la investigación feminista. Por ello, según Miller y Boulton, (2007) una de las estrategias que utiliza la investigación feminista para reducir las diferencias de poder entre investigadores/as y participantes es el empoderamiento a lo largo del proceso de investigación.

Los compromisos o responsabilidades que asumimos la investigación también requieren de reflexión inicial y durante el proceso. Hasta el punto de que las personas participantes nos interpelen y nos encomienden tareas y responsabilidades en el curso de la actividad de investigación y se puedan generar tensiones y dilemas de poder. Estos aspectos han de ser conocidos como posibles cuando planteamos la investigación feminista.

“durante todo el proceso nos fuimos sintiendo interpeladas por el propio colectivo, lo que nos lleva a pensar los puntos sobre la asunción de responsabilidades; la valoración y el respeto de las agencias de todas las subjetividades; así como la puesta en juego de las dinámicas de poder" (Araiza y González, 2017: 75).

La consideración de con quién realizaremos la investigación requiere necesariamente que contemplemos también el principio de interseccionalidad del 
género con otras categorías sociales como edad, clase, raza/etnia, sexualidad, discapacidad. Hay que atender a la diversidad dentro de cada una de ellas. Es un problema considerar, por ejemplo, que las mujeres como grupo y/o los hombres como grupo (sus actitudes, preferencias, necesidades, conductas y conocimientos) son lo mismo. Hay que tener en cuenta que las diferencias entre mujeres y hombres no se mantienen a través de las culturas.

\section{4. ¿Cómo?}

El diseño metodológico de la investigación feminista requiere repensar metodologías y métodos conforme a objetivos y principios y valores feministas. Hay una tendencia a pensar que la investigación feminista rechaza los métodos cuantitativos. Esta idea se genera en el rechazo y cuestionamiento del razonamiento científico hegemónico, las prácticas de investigación pretendidamente imparciales de investigadores abstractos y descontextualizados y el hecho de que tiende a "objetivizar" a "sujetos" de investigación. No obstante, al igual que se están reinventando las metodologías cualitativas de forma que "se empujan" sus límites tradicionales, como veremos más adelante en este trabajo, también los métodos cuantitativos se están revisando y doblegando a las epistemologías feministas (Else-Quest y Hyde, 2016a). En este sentido, las metodologías feministas son muy variadas, y no hay opciones metodológicas rechazables, solo el uso que hacemos de ellas y al servicio de qué las ponemos pueden determinar su aporte a este campo de conocimiento.

\subsubsection{Decisiones sobre los métodos de investigación feministas}

De acuerdo con las epistemologías, las preguntas de investigación y los objetivos es preciso tomar decisiones sobre los métodos. La investigación feminista ha ido consolidando formas de investigación que merecen ser denominadas "métodos feministas". La siguiente figura muestra algunos de estos métodos que se abren paso como nuevas formas de hacer ciencia y que hemos podido recabar de la revisión de literatura llevada a cabo. Los artículos abordados se centran especialmente en métodos cualitativos e investigación acción feminista. Los métodos cualitativos convencionales se muestran caracterizados por nuevos rasgos y procesos dando paso a métodos de investigación emergentes de corte cualitativo y feminista como los que ampliamos a continuación

\subsubsection{Métodos etnográficos feministas.}

Concretamente, y a diferencia de otros enfoques la etnografía feminista tiene su propia genealogía, se inicia en los años 80 de la mano de antropólogas feministas (Visweswaran, 1997). Entre los métodos cualitativos los métodos etnográficos se han usado en la investigación feminista y de género para profundizar en 
el sentido cultural de las experiencias de vida particulares. Una importante parte de la tradición etnográfica se ha centrado en textualizar las experiencias que se desarrollan durante el trabajo de campo. La mirada feminista ha puesto el acento también en otras experiencias sensoriales que van más allá de la textualización o escritura de la observación. Así, para conocer las formas culturales de vida han puesto de relevancia otros sentidos más allá de la vista o de la observación y hablan de una "etnografía de contactos" (Pérez-Bustos, Tobar-Roa y MárquezGutiérrez, 2016; Howes 2014). También se ha delimitado el carácter de las "autoetnografías feministas" (Smailes, 2014: 60). La revisión de la literatura más actual muestra la relevancia de la "etnografía virtual feminista" como método reciente (Astudillo-Mendoza, Figueroa-Quiroz y Cifuentes-Zunino, 2020).

\subsubsection{Métodos fenomenológicos feministas}

La tradición fenomenológica feminista se inicia con publicaciones en EE.UU y Alemania hace veinte años (Young, 2005). Según López (2014) esta perspectiva no consiste en aplicar la fenomenología a los estudios de género, sino en interrelacionar dos tradiciones de pensamiento. Por ello, estas contribuciones no se ocupan de construir una nueva teoría feminista desde la fenomenología, sino de desarrollar con ella conceptos y métodos que permitan comprender el sentido de las desigualdades de género. De acuerdo con Simms y Stawarska (2013) la fenomenología es feminista, siempre y cuando incluya preguntas relacionadas con la experiencia de género y la diferencia sexual dentro de su campo.

El aporte feminista a la fenomenología recae en el análisis estructural más amplio de la situación en la que la experiencia vivida se desarrolla. Desde un punto de vista metodológico y siguiendo a Simms y Stawarska (2013) destacamos que la fenomenología feminista: a) Trabaja estrechamente con descripciones en primera persona sobre experiencias humanas específicas y se empeña en iluminar la complejidad de investigar los mundos de las personas participantes. $\mathrm{Su}$ objetivo es la profundidad y la comprensión de la condición humana, b) Empuja a los investigadores e investigadoras a cuestionar sus suposiciones conceptuales y los supuestos que sustentan sus teorías de investigación y sus prácticas y abre el campo a nuevas formas de entender lo que se está investigando.

En esencia, la práctica de la investigación feminista parte de comprender la experiencia humana como experiencia "encarnada", intersubjetiva y enredada en significados personales y culturales. Las experiencias de las personas que participan en la investigación tienen que ser tratadas con interés, respeto y empatía, pero siempre interpretadas desde una perspectiva crítica. La fenomenología feminista se concreta en métodos de narrativos anidados o métodos de producciones textuales coproducidas. En el siguiente ejemplo podemos observar las exigencias que suponen estas nuevas formas de construcción del conocimiento científico y el grado de compromiso que exige a quien participa:

"En otros casos, las altas exigencias que implica la elaboración conjunta de una producción narrativa (tiempo, participación, redacción, lecturas) y el nivel de compromiso social o político de las personas participantes hicieron 
que sus contribuciones fueran menores y más de corrección estilística; en otros casos jugó en contra el fin de conseguir un texto que pudiera ser reconocido a nivel teórico lo que afectó a aquellos/as participantes con menos recursos lingüísticos y habilidades escritas para poder adentrarse en la coconstrucción del relato” (Pérez, Valderrama, y Álvarez, 2017: 2017).

En este sentido, la relación asimétrica y de poder entre investigadores/as y personas participantes siempre ha supuesto un reto para la investigación feminista, que afecta especialmente a los procesos metodológicos relacionados con la recogida de información:

"No en todos los casos se consiguió de manera sostenida el compromiso de las personas en cada una de las sesiones; algunas participantes acostumbradas a una forma hegemónica de investigación no siempre hicieron suyo el proceso porque se mantenían en una suerte de posición de "informante" " (Pérez, Valderrama, y Álvarez, 2017).

\subsubsection{Métodos fenomenográficos feministas}

Desde una perspectiva feminista Hazel, Conrad y Martin (1997), alertaban de la falta de mujeres en la investigación fenomenográfica. El principal reto metodológico para la fenomenografía es la medida de la "variedad" de experiencias individuales y evitar categorías de análisis de la experiencia excesivamente abstractas y lejanas a cómo las personas que participan en la investigación las representan. Por ello, el reto es la búsqueda de estrategias de autorrepresentación en la investigación y con capacidad suficiente como para captar la variedad de experiencias.

Actualmente, los métodos visuales son una alternativa para la investigación feminista. Aunque la investigación ha venido usando los métodos visuales como parte del proceso de generar evidencia para explorar preguntas de investigación (como fotografías, diagramas, mapas relacionales, cronogramas, autorretratos, collages, películas, dibujos, novelas gráficas, foto-diarios) (Rose, 2013), lo cierto es que, en menor medida, se han usado bajo supuestos de investigación feminista (Allen, 2009), o se usan junto con producciones discursivas a través de técnicas como la fotovoz (Robinson-Keilig, et al., 2014).

\subsubsection{Métodos biográficos feministas}

La investigación feminista está dando un nuevo giro al método. Ya lo habían puesto en tensión incorporando en las investigaciones biográficas el cuerpo. Al incorporar el cuerpo en la investigación se abren nuevos desafíos metodológicos (Pujol, Montenegro y Balasch, 2003). Silva, Barrientos y Espinoza (2013) emplean el método de mapas corporales para generar un entramado en la biografía de cada persona que contemple el sentido y la significación del sí mismo y su corporalidad expresada como lenguaje. Estos autores y autoras teniendo en cuenta las experiencias biográficas encarnadas, introducen los mapas corporales en la 
investigación biográfica para hacer emerger significados y discursos encarnados en un cuerpo protagonista de la biografía.

En el mismo sentido, la revisión de la literatura muestra estudios actuales con biografías colectivas (Gannon, y Gonick, 2019). En estudios sobre abuso infantil, agresión sexual y violencia de género, las biografías colectivas suponen un giro metodológico y exigen toma de decisiones rápidas por parte de quien investiga con altos grados de sensibilidad que afectan al trabajo de campo y a la recogida de información. Así, lo exponen Fraser y MacDougall (2017):

"Las narradoras seleccionan (conscientemente y de otra manera), qué historias contarán, cómo las contarán” (...) "Por ejemplo, hay que armarse de coraje y confianza para que las participantes de In the Name of Love le revelaran a un investigador desconocido incidentes de abuso infantil, agresión sexual y violencia doméstica” (2017: 244)

\section{5. ¿Para qué?}

Cuando nos planteamos el diseño de una investigación feminista hay que pensar también en las consecuencias sociales, educativas, psicológicas...de la investigación. En definitiva, ¿cómo vamos a contribuir con la investigación a la justicia social o a la igualdad de oportunidades para las mujeres? La respuesta a esta pregunta es la que marca la diferencia conceptual de lo que implica plantearse una investigación feminista frente a otras formas de investigación en ciencias sociales. Para Kaur y Nagaich (2019: 4) esto se concreta en retratar y sacar a la luz los cambios estructurales necesarios en la sociedad:

El análisis feminista no puede pretender hablar por todas las mujeres, sin embargo, ofrecerá nuevos datos basados en las realidades de las experiencias de las mujeres y retratará activamente los cambios estructurales dentro del mundo social.

El para qué se investiga es fundamental en la investigación feminista, ya que la propia investigación lleva implícito el sentido y el aporte. La investigación feminista tiene la obligación de que se visibilice: a) la relevancia del estudio en términos de cambios estructurales de la desigualdad (educativa, social, política...), b) la identificación de beneficios concretos/as para las mujeres y c) el impacto en la acción social inmediata o futura.

\subsection{Procesos visibles e invisibles de investigación: "Huellas" en la difusión de los textos científicos}

La investigación feminista tiene la particularidad de que descubre "las cocinas" de la investigación (Biglia y Vergés, 2016). Los procesos por los que transcurre la investigación se destapan en un intento de responder a los procesos 
de reflexividad como uno de los principales elementos de calidad y validez de los resultados.

En la redacción de los textos científicos que recogen investigación feminista se desvelan las formas de interrogar y examinar cómo las características sociales propias han intervenido o informado el proceso de investigación (HesseBiber, 2014). Esto da lugar a una escritura concreta del texto científico donde se verbalizan y hacen explícitos procesos de investigación que no tienen lugar en la estructura tradicional/hegemónica de un informe de investigación (sea un artículo, una comunicación o un informe técnico). Los procesos que en la investigación tradicional quedan ocultos, en la investigación feminista salen a la luz para rendir cuentas del proceso reflexivo, durante el cual las investigadoras e investigadores desarrollan una "conciencia aguda" (Olesen, 1994:135) sobre cómo diferentes elementos vitales, contextuales modulan y afectan la experiencia de investigación. Esta conciencia aguda y su rastro en los resultados de investigación se convierten en la garantía de calidad del conocimiento situado que se produce.

La incorporación de estos procesos en el texto científico y su impacto en los resultados de investigación y en las decisiones metodológicas tienen que pasar previamente por ser registrados y sistematizados al igual que la información objeto de estudio. Esto implica que, tanto en el proyecto como en los informes derivados de la investigación, se plasmen aspectos internos que afectan a dudas propias de los procesos de investigación y que en la feminista se explicitan:

"Nos reconocemos como participantes del grupo, haciendo explícito que nuestra incorporación a este precedió a la idea de estudiar/nos. Al tomar esta decisión, cuestionamos los límites entre nuestras diferentes posiciones surgiendo la reflexión acerca de si podemos -o no-, habitar la investigación de manera fluida desde nuestra propia experiencia de participación" (AstudilloMendoza, Figueroa-Quiroz y Cifuentes-Zunino, 2020: 245).

Las emociones, sensaciones, reflexiones del propio investigador o investigadora se sitúan un mismo plano crítico que las que provienen de las personas participantes. Estas emociones se verbalizan y quedan registradas al unísono en la redacción del texto científico mostrando cómo afectan a los resultados. Los resultados de la investigación feminista quedan imbuidos de valores y perspectivas personales siendo también el producto de procesos éticos.

\section{DESAFÍOS METODOLÓGICOS DE LA INVESTIGACIÓN FEMINISTA}

Los desafíos metodológicos que plantea la investigación feminista están relacionados fundamentalmente con las epistemologías. Las derivaciones metodológicas hacen referencia a: a) la atención a las categorías sociales que van más allá del género (etnia, edad, orientación sexual...), b) la incorporación del rol reflexivo de la investigadora o el investigador en el proceso de investigación 
y el desarrollo de una conciencia aguda de los procesos y contextos, c) la consideración de la diversidad de experiencias de vida. Lo que conlleva una preocupación por las consecuencias sociales de la investigación científica y, por tanto, de sus métodos; orientados hacia la transformación de las estructuras de poder y desigualdad en la búsqueda de justicia social, y d) el papel de las emociones en la investigación.

\subsection{Desafío metodológico 1. La medida de la interseccionalidad.}

Nicole Else-Quest y Janet Hyde (2016a, 2016b) definen de forma operativa la interseccionalidad como: (1) Un reconocimiento de que todas las personas se caracterizan simultáneamente por múltiples categorías, incluyendo, por ejemplo, género, raza y etnia, clase y orientación sexual; estas múltiples categorías sociales están interconectadas o entrelazadas, de modo que la experiencia de cada categoría social está vinculada a las otras categorías, (2) el reconocimiento de la desigualdad o el poder resultan esenciales para el análisis interseccional, (3) estas categorías son propias de las personas y las caracterizan y su significado puede ser fluido y dinámico. Si bien no existe una definición consensuada de interseccionalidad, creemos de acuerdo con las autoras que estas suposiciones comunes proporcionan una caracterización suficientemente inclusiva y apropiadamente específica del concepto para formar una definición de este.

La interseccionalidad como desafío metodológico se presta a ser incorporada en la investigación cualitativa ya que los procesos son más sensibles a interpretar las posiciones sociales de las personas participantes y cómo ellas las viven, representan y experimentan. Los datos de perfil y condiciones personales de las personas participantes también están más presentes en la recogida de información y en el análisis dependiendo especialmente de una comprensión del contexto social, político e histórico en que las experiencias de estas personas están teniendo lugar. Por lo tanto, estas "ubicaciones" de intersección ayudan a comprender las experiencias vitales y los fenómenos sociales que estemos estudiando, favoreciendo así, el análisis de estructuras sociales desiguales.

En investigación cuantitativa el reto metodológico recae en la preocupación sobre la compatibilidad de la interseccionalidad con los métodos cuantitativos. Ya que, las dudas parten de las cuestiones epistemológicas que equiparan los métodos cuantitativos con una epistemología positivista y los métodos cualitativos con una epistemología construccionista social (Sprague, 2005). No obstante, ya hay estudios que revisan esta posibilidad y lanzan propuestas para aplicarlo y sistematizarlo desde la investigación cuantitativa (Else-Quest y Hyde, 2016a, 2016b).

Concretamente, en investigación cuantitativa es relativamente fácil incorporar variables de perfil que reflejen la pertenencia de las personas que participan a diferentes categorías sociales. No obstante, en esta investigación los resultados deben ser interpretados en el marco de un contexto social, histórico y/o que explique la desigualdad estructural si no perderá sentido y fuerza como inves- 
tigación feminista o de género. También el contexto social y los significados atribuidos a las diferentes categorías sociales pueden estar presentes en la propia definición de variables consideradas y, en consecuencia, en la medida. Por ejemplo, hay escalas diseñadas para captar cuantitativamente las experiencias de las niñas y las mujeres, tales como la Escala de Conciencia del Cuerpo Objetivada (McKinley y Hyde, 1996).

Metodológicamente trasladar la idea de reconocimiento de la desigualdad y el poder supone situar la atención en el análisis de datos y en la interpretación. El foco de atención de la investigación cuantitativa para incorporar la interseccionalidad está también en el análisis de datos atendiendo a las diferencias significativas entre miembros de esas categorías sociales.

En cuanto a que la investigación para que sea interseccional tiene que obedecer al carácter fluido y dinámico de pertenencia a estas categorías sociales (Else-Quest y Hyde, 2016a) puede tener derivaciones metodológicas para la recogida de datos cualitativos (donde las personas participantes puedan elaborar retrospectivas o prospectivas) e incluso podemos recoger datos de las mismas personas en otras situaciones vitales y contextos de tal manera que se pueda observar cómo las categorías sociales fluctúan, cambian, evolucionan.

\subsection{Desafío metodológico 2. Reflexividad como criterio de rigor}

Diversos términos se utilizan para referirse a este criterio que metodológicamente consideramos emergente: subjetividad crítica, subjetividad transformadora y reflexividad crítica. En esencia, se refiere al reconocimiento de que la investigadora o el investigador no es una figura aislada del contexto, grupo, cultura... que intenta comprender y representar, sino que forma parte de él.

El proceso de investigación se convierte en un espacio para la reflexión (la investigación tiene que ser reflexiva). Según Denzin (1997: 27) la reflexividad hace referencia a la forma en que nuestra subjetividad como investigadores/as se "enreda en las vidas de los demás". La reflexividad ha sido objeto de controversias y atención especial para la investigación feminista (Doucet y Mauthner, 2006). Para estas autoras, la mayoría de los investigadores e investigadoras feministas reflexionan, reconocen y documentan abiertamente su ubicación social, los roles que desempeñan en la cocreación de datos y en la construcción de conocimiento y las formas en que nuestras respuestas emocionales ante las personas pueden dar forma a nuestras interpretaciones de sus relatos. Estas autoras revisan el concepto de reflexividad y la entienden como "reflexividad en retrospectiva", es decir, una forma de ver la investigación y el conocimiento producido como un proceso continuo y abierto que cambia a medida que las personas que investigan revisan sus datos y abren los resultados a nuevos investigadores/as que analicen viejos conjuntos de datos.

La reflexividad también se ha relacionado con la "responsabilidad", como una forma de explicar el conocimiento producido (Doucet y Mauthner, 2006). Desde este punto de vista, la reflexividad hace referencia a una reflexión activa 
sobre las influencias personales, interpersonales, institucionales, emocionales, teóricas, epistemológicas de nuestra investigación y procesos interpretativos. Según Mauthner y Doucet (1998) existe un amplio reconocimiento de que la interpretación de los datos es en sí misma un ejercicio reflexivo a través del cual se elaboran significados en lugar de encontrarlos. La manera en cómo han intentado operativizar la reflexividad ha consistido en elaborar una hoja de trabajo con dos columnas durante el análisis de datos. En una de las columnas se especifica el contenido de los relatos o transcripciones de entrevistas o fragmentos de diarios en otra se anotan las reacciones e interpretaciones de la persona que investiga lo que permite incorporar la reflexiones a los procesos de análisis.

El trabajo más actual de Linabary y Hamel (2017), propone el uso de preguntas "reflexivas" que invitan a los participantes a atribuir significado a sus respuestas. Estas se utilizan para garantizar que la reflexividad esté incorporada en el diseño, implementación y en la recopilación de datos y la reflexión posteriores al estudio. Algunas de ellas hacen referencia a pedir a los encuestados que reflexionen sobre la experiencia de la entrevista y atribuyan significado a sus experiencias o preguntarnos si hay o ha habido oportunidades (dadas y aprovechadas) para compartir el poder y reflexionar críticamente sobre él.

"En lugar de ver la reflexividad como "una casilla metodológica que hay que marcar", ser incómodamente reflexivo nos invita a "resistir los regímenes disciplinarios de la verdad y tratar de poner en primer plano continuamente el funcionamiento del poder en las investigaciones y representaciones de uno" (Dosekun 2015: 2, cit. en Bishop, 2018: 246).

Este proceso de reflexionar sobre las relaciones de poder en la investigación fortalece la validez de los resultados (Astudillo-Mendoza, Figueroa-Quiroz, y Cifuentes-Zunino, 2020, Yarbrough, 2019). Por ello, se impone como criterio de rigor prioritario en la investigación feminista actual.

\subsection{Desafío metodológico 3. Reinvención de métodos y técnicas convencionales para respetar la voz y prácticas éticas en torno a la agencia}

La investigación feminista se enfrenta al desafío de reinventar metodológicamente los métodos y técnicas convencionales de investigación en ciencias sociales (Moss, 2007: 374). La reinvención de estos métodos y técnicas se orientan a realzar la voz, es decir, hacer hincapié en quién habla, a quién, desde qué propósitos construye una determinada realidad. Según Pamela Moss (2007: 372), en la recogida de información se usan técnicas y estrategias novedosas que integran representaciones visuales en el proceso de investigación. Por ejemplo, la introducción del video en la recogida de datos, en el análisis y en la presentación de los resultados de investigación, combinación de fotografías, sesiones informativas y "cuentacuentos" (storytelling), fotovoz, presentación de video clips o producciones digitales (Bishop, 2018). 
En numerosas ocasiones la investigación feminista al velar por la voz y la agencia de quien participa plantea quiebras en las prácticas éticas convencionales. Por ejemplo, el difícil equilibrio entre la agencia y el anonimato en la investigación. Gordon (2019) se centra en una investigación en Bihar, India, donde muchos de los encuestados rechazaron la idea del anonimato. Y es que como afirma la autora, mantener una ética feminista del cuidado y cumplir con el compromiso feminista de dar voz a las mujeres requiere un enfoque en la agencia de las mujeres en el proceso de toma de decisiones en torno a la ética:

"El anonimato puede ser de suma importancia en muchos entornos de investigación, particularmente en la investigación feminista o en la investigación donde la participación y los hallazgos pueden conducir a represalias para los participantes y amenazas a su seguridad. Sin embargo, el anonimato tiene el potencial de contradecir la importancia en la investigación feminista de que las mujeres sean dueñas de su propia narrativa y cuenten su historia mientras afirman su propia identidad. El anonimato también puede obstaculizar fundamentalmente los objetivos emancipatorios y políticos de la investigación feminista para crear espacios de resistencia y cambio transformador" (Gordon, 2019: 551).

\subsection{Desafío metodológico 4. Emociones. "Investigaciones emocionalmente implicadas", "vivir el campo" como investigadores/as.}

García-Dauder y Ruiz-Trejo (2018) recogen la idea de Blakely (2007) sobre las "investigaciones emocionalmente implicadas". Hasta el momento las emociones no han formado parte de los procesos de investigación y, sin embargo, las experiencias que tenemos como investigadores e investigadoras tienen un carácter "encarnado", es decir, tienen que ver con nuestros "cuerpos" y con cómo experimentamos los diferentes momentos de la investigación. Especialmente, cuando se trata de investigaciones sobre temas difíciles y delicados (acoso, violencia...). De ahí que, los Estudios de las Emociones y la Afectividad ocupen un lugar muy productivo desde el que pensar en todas aquellas regulaciones que exceden lo social y lo discursivo (López, 2014). Según López (2014), centrarnos solo en un modelo discursivo en la investigación genera insatisfacciones al entender que la realidad social no se limita al discurso lo que implica que es necesario dar un "giro emocional" y "afectivo". Este enfoque teórico y metodológico reconoce que en las dinámicas sociales (entre las que se encuentra la propia investigación) están presentes fuerzas del orden de lo corporal tanto como discursivas. El planteamiento de la investigación feminista es que hay diferencias entre el concepto de emociones y el de afectos. El afecto está más relacionado con la relación cultural que mantenemos con los/as otros/as. Sara Ahmed plantea las emociones en términos de ideas y valores. Es decir, como juicios sobre las cosas (Schmitz y Ahmed, 2014). Y lo ejemplifica "odiar o temer es tener un 
juicio sobre una cosa a medida que se acerca" (2014: 101). Pero también enfoca el concepto en reacciones corporales que surgen sin pensar (viscerales) y que denomina como "sensación". Usar el término sensación atiende a esa respuesta visceral. Estudios actuales se ocupan de los afectos como metodología (Åhäll, 2018).

Los estudios feministas han estado preocupados desde hace décadas por la conexión entre sentimientos e investigación constituyendo todavía hoy un reto metodológico. Blakely (2007) hace referencia al "investigador/a investigado/a" precisamente para hacer hincapié en la necesidad de recabar información sobre nuestras emociones en las diferentes etapas y procesos por lo que transcurre la investigación. Esta investigación emocionalmente implicada se guía por una ética del cuidado que conlleva, responsabilidad con respecto al tema o cuestión que se está investigando, cuidado y respeto hacia las personas que participan, hacia los materiales e informaciones (narrativas, notas de investigación...), atención a las emociones y la afectividad tanto de la persona o equipo investigador como de los/as participantes.

Blakely (2007: 60) ofrece pautas para operativizar las emociones en el proceso de investigación, como, por ejemplo; anotar sentimientos durante y después del proceso de investigación en notas de campo o en un diario personal, o dar un lugar a los sentimientos incluyéndolos en el epílogo de un trabajo terminado. En este proceso no se trata solo de visibilizar emociones sino de conectarlas con el conocimiento producido. Algunas preguntas que nos pueden ayudar a capturarlas y sistematizarlas pueden ser: ¿Qué despertó mis emociones y cuáles fueron esos sentimientos? ¿Qué sucedió durante el proceso de investigación que más me afectó y cómo me hizo sentir? ¿Todavía siento lo mismo? ¿Algunas de las emociones se quedaron conmigo después de las entrevistas o el estudio, y por cuánto tiempo? ¿Qué emociones y por qué?

"Las emociones son importantes fuentes de conocimiento incorporado que apuntan a áreas de información y posible conversación y análisis futuros. En las entrevistas en nombre del amor, los participantes a menudo se reían, a veces con humor, otras veces cínicamente o con autodesprecio, por lo que habían estado dispuestos a hacer por amor. Algunas mujeres lloraron, otras mujeres se enojaron, se entristecieron o una mezcla (diferente) de emociones. Descifrar lo que estas emociones significaban para el narrador (no solo el investigador) fue parte del desafio" (Fraser y MacDougall, 2017: 245)

El trabajo de Jackson (2011) sobre la identidad y la ciudadanía de mujeres inmigrantes es un buen ejemplo para observar cómo la epistemología feminista reta a los métodos de investigación. En este caso, Jackson (2011) plantea la idea de "vivir el campo" para sustituir la expresión tradicional de la fase de "trabajo de campo" en la investigación. En este sentido, las vivencias y la conexión entre investigadora y mujeres inmigrantes se recaban como elementos importantes del estudio. Así, esta investigadora incorpora narrativas sobre el trabajo de campo donde recoge las emociones que los procesos de negociación de acceso y contac- 
to con el colectivo le producen, hace hincapié en la empatía con algunos grupos de mujeres y la experiencia compartida.

\section{CONCLUSIÓN}

La investigación feminista sigue los principios de las epistemologías feministas desencadenando otras formas de hacer ciencia, asumiendo la reinvención del propio proceso de investigación, la revisión de los métodos convencionales a emplear, abriéndose al impacto de las emociones y ejercitando de forma práctica la reflexividad. Los principios de las epistemologías feministas no son fácilmente trasladables a la práctica de la investigación. De ahí que, este trabajo concluya ofreciendo los principales elementos metodológicos que la definen y que caracterizan su diseño e implementación. Las pautas para el diseño de la investigación feminista se extraen de una revisión de bibliografía actualizada y referente que permiten su uso aplicado para investigadores o investigadoras tanto noveles como consolidados/as. Cada elemento del diseño de investigación parte del análisis de un número amplio de contribuciones y referentes en la materia y en la propia experiencia investigadora poniendo a disposición de la academia una breve guía didáctica fundamentada y sintética de pasos ilustrados.

El trabajo apunta también hacia los principales retos que la investigación feminista presenta en la actualidad y que nos desafían como especialistas en metodologías de la investigación abriendo nuevos caminos por recorrer. Se ofrece así, un conjunto de líneas de debate abierto en la comunidad científica y que afectan directamente a la práctica de este tipo de investigación tanto como a su enseñanza.

\section{REFERENCIAS BIBLIOGRÁFICAS}

AGEE, J. (2009): "Developing qualitative research questions: a reflective process, International", Journal of Qualitative Studies in Education, 22, 4, pp. 431-447.

ÅHÄLL, L. (2018): “Affect as methodology: Feminism and the politics of emotion", International Political Sociology, 12, 1, pp. 36-52.

ALLEN, L. (2009): "Snapped: Researching the sexual cultures of schools using visual methods", International Journal of Qualitative Studies in Education, 22, 5, pp. 549561

ASTUDILLO-MENDOZA, P. A., FIGUEROA-QUIROZ, V. A., y CIFUENTES-ZUNINO, F. (2020): "Navegando entre mujeres: La etnografía digital y sus aportes a las investigaciones feministas", Investigaciones Feministas, 11, 2, pp. 239-250.

ASTUDILLO-MENDOZA, P. A., FIGUEROA-QUIROZ, V. A., y CIFUENTES-ZUNINO, F. (2020): "Navegando entre mujeres: La etnografia digital y sus aportes a las investigaciones feministas", Investigaciones Feministas, 11, 2, pp. 239-250.

ATTIA, M., y EDGE, J. (2017): "Be (com) ing a reflexive researcher: A developmental approach to research methodology", Open Review of Educational Research, 4(1), pp. 33-45. 
BARATA, P.; HUNJAN, S. Y LEGGATT J. (2005): "Ivory tower? Feminist women's experiences of graduate school Women's Studies", International Forum, 28, 2-3, pp. 232-246.

BIGLIA, B. y VERGÉS-BOSCH, N. (2016): “Cuestionando la perspectiva de género en la investigación”, Revista d'Innovació i Recerca en Educació, 9, 2, pp.12-29.

BIGLIA, B., y I MARTÍ, J. B. (2017): "DIY: Towards feminist methodological practices in social research", Annual Review of Critical Psychology, 13, pp. 1-16.

BISHOP, S. (2018): "Fetishisation of the "offline" in feminist media research", Feminist Media Studies, 18, 1, pp.143-147.

BLAKELY, K. (2007): "Reflections on the role of emotion in feminist research", International Journal of Qualitative Methods, 6, 2, pp. 59-68.

COOKY, C., LINABARY, J. R., y CORPLE, D. J. (2018): "Navigating Big Data dilemmas: Feminist holistic reflexivity in social media research", Big Data \& Society, 5, 2.

DENZIN, N. (1997): Interpretive Ethnography: Ethnographic Practices for the 21st Century, Thousand Oaks, Sage.

DÍAZ, A. A., y GARCÍA, R. G. (2017): "La Investigación Activista Feminista. Un diálogo metodológico con los movimientos sociales", EMPIRIA. Revista de Metodología de las Ciencias Sociales, 38, pp. 63-84.

DOUCET, A., y MAUTHNER, N. S. (2006): "Feminist methodologies and epistemology", en The Handbook of 21st Century Sociology, Thousand Oaks, CA, Sage Publications, pp. 36-42.

ELSE-QUEST, N. M. y HYDE J., S. (2016b): "Intersectionality in Quantitative Psychological Research: II", Methods and Techniques, Psychology of Women Quarterly, 40, 3, pp. 319-336.

ELSE-QUEST, N. M. y HYDE JANET, S. (2016a): “Intersectionality in Quantitative Psychological Research: I", Theoretical and Epistemological Issues, Psychology of Women Quarterly, 40, 2, pp.155-170.

FALCONER, E. (2017): "Moments of collusion? Close readings of affective, hidden moments within feminist research", Women's Studies International Forum, 61, pp. $75-80$.

FRASER, H., y MACDOUGALL, C. (2017): "Doing narrative feminist research: Intersections and challenges", Qualitative Social Work, 16, 2, pp. 240-254.

GANNON, S., y GONICK, M. (2019): "Collective biography as a feminist methodology", en Strategies for Resisting Sexism in the Academy, Palgrave Macmillan, Cham, pp. 207-224.

GARCÍA, N. y MONTENEGRO, M. (2014): "Re/pensar las Producciones Narrativas como propuesta metodológica feminista", Athenea Digital, 14, 4, pp. 63-88.

GARCÍA-DAUDER, S. y RUIZ TREJO, M. (2018): "El papel de las emociones en la investigación”, Ponencia presentada al XII Congreso Iberoamericano de Ciencia, Tecnología y Género, celebrada en Bilbao, 18-20 de Julio, inédita.

GORDON, R. (2019): "Why would I want to be anonymous? Questioning ethical principles of anonymity in cross-cultural feminist research", Gender \& Development, 27, 3, pp. 541-554.

HAZEL, E.; CONRAD, L. y MARTIN E. (1997): Issues in gender and phenomenography, AARE Annual Conference, Brisbane.

HESSE-BIBER, S. N. (2014): "Feminist approaches to in-depth interviewing", en Feminist Research Practice, London, UK, Sage, pp.182-232 
HOWES, D. (2014): "El creciente campo de los estudios sensoriales", Revista latinoamericana de estudios sobre cuerpos, emociones y sociedad, 6, 15, pp.10-26.

JACKSON, L. (2011): "Mixed Methodologies in Emotive Research: Negotiating Multiple Methods and Creating Narratives in Feminist Embodied Graduate", Journal of Asia-Pacific Studies, 7, 2, pp. 46-6.

JIMÉNEZ-CORTÉS, R. (2019): Investigación educativa con perspectiva de género, Aula Magna, McGrawHill.

KAPLAN, G. (1995): "Feminist methodology is it a fact or fiction?", Bulletin de Méthodologie Sociologique, 46, pp. 88-98.

KAUR, R., y NAGAICH, S. (2019): "Understanding feminist research methodology in social sciences", SSRN Electronic Journal, disponible en: <https://papers.ssrn.-com/ sol3/papers.cfm?abstract_id=3392500> [consulta: 12-11-2019]

LEAVY, P., y HARRIS, A. (2019): Contemporary feminist research from theory to practice, New York, NY, Guilford Press.

LINABARY, J. R., y HAMEL, S. A. (2017): "Feminist online interviewing: engaging issues of power, resistance and reflexivity in practice", Feminist review, 115, 1, pp. 97-113.

LÓPEZ, H. (2014): "Emociones, afectividad, feminismo", en Cuerpo y afectividad en la sociedad contemporánea, México, UAM-A, pp. 257-275

MAUTHNER, N.S. y DOUCET, A. (1998): "Reflections on a Voice Centred Relational Method of Data Analysis: Analysing Maternal and Domestic Voices", en Feminist Dilemmas in Qualitative Research: Private Lives and Public Texts, London, Sage; pp. 119-144

MCKINLEY, N. M., y HYDE, J. S. (1996): "The objectified body consciousness scale: Development and validation", Psychology of Women Quarterly, 20, pp. 181-216.

MILLER, T., y BOULTON, M. (2007): "Changing constructions of informed consent: qualitative research and complex socia worlds". Social Science and Medicine, 65,11, pp. 2199-2211

MOSS, P. (2007): "Emergent methods in feminism research", en Handbook of feminist research, Theory and praxis, London, Sage Publications, pp. 371-390.

NAZNEEN, S.; DARKWAH, A y SULTAN, M. (2014): "Researching women's empowerment: Reflections on methodology by southern feminists", Women's Studies International Forum 45, pp. 55-62

OLESEN, V. L. (1994): "Feminist qualitative research", en Hanbook of Qualitative Research, Thousand Oaks, CA, Sage, pp. 158-174

PÉREZ, L. T., VALDERRAMA, C. G., y ALVAREZ, C. (2017): "Las producciones narrativas como metodología de investigación feminista en Psicología Social Crítica: Tensiones y desafíos", Psicoperspectivas, 16, pp. 20-citation_lastpage.

PÉREZ-BUSTOS, T.; TOBAR-ROA, V. y MÁRQUEZ-GUTIÉRREZ S. (2016): "Etnografías de los contactos. Reflexiones feministas sobre el bordado como conocimiento", Antipod. Rev. Antropol. Arqueol. 26, pp. 47-66.

PUJOL, J.; MONTENEGRO, M. y BALASCH, M. (2003): "Los límites de la metáfora Lingúística: implicaciones de una perspectiva corporeizada para la práctica investigadora e interventora", Política y Sociedad, 40, 1, pp. 57-60.

RAYAPROL, A. (2016): "Feminist research: Redefining methodology in the social sciences", Contributions to Indian Sociology, 50, 3, pp. 368-388.

ROBINSON-KEILIG, R. A., HAMILL C., GWIN-VINSANT A., DASHNER M. (2014): "Feminist pedagogy in action: Photovoice as an experiential class Project", Psychology of Women Quarterly, 38, pp. 292-297. 
ROSE, G. (2013): “On the relation between 'visual research methods' and contemporary visual culture", The Sociological Review, 62, pp. 24-46.

RUAN, N. (2020): "Interviewing elite women professors: Methodological reflections with feminist research ethics", Qualitative Research, 1-16.

SCHMITZ, S. y AHMED, S. (2014): “Affect/Emotion: Orientation Matters. A Conversation between Sigrid Schmitz and Sara Ahmed", Freiburger Zeitschrift für Geschlechter Studien, 20, 2, pp. 97-108.

SILVA, J., BARRIENTOS, J., y ESPINOZA-TAPIA, R. (2013): “Un modelo metodológico para el estudio del cuerpo en investigaciones biográficas: los mapas corporales", Alpha (Osorno), 37, pp. 163-182.

SIMMS, E. M. y STAWARSKA, B. (2013): "Introduction: Concepts and Methods in Interdisciplinary Feminist Phenomenology”, Janus Head, 13, 1, pp. 6-16.

SMAILES, S. (2014): "Negotiating and navigating my fat body-feminist autoethnographic encounters", Athenea Digital, 14, 1, pp. 49-61.

SPRAGUE, J. (2005): Feminist Methodologies for Critical Researchers, Bridging Differences, UK, Rowman Altamira.

VAN DER HAAR, M., y VERLOO, M. (2016): "Starting a conversation about critical frame analysis: reflections on dealing with methodology in feminist research", Politics \& Gender, 12, 3.

VISWESWARAN, K. (1997): "Histories of Feminist Ethnography", Annual Review of Antrhopology, 26, pp. 591-621

WALTERS, R. (2020): "Relinquishing control in focus groups: the use of activities in feminist research with young people to improve moderator performance", Qualitative Research, 20, 4, pp. 361-377.

WIGGINTON, B., y LAFRANCE, M. N. (2019): "Learning critical feminist research: A brief introduction to feminist epistemologies and methodologies", Feminism \& Psychology, pp.1-17

YARBROUGH, D. (2020): "Nothing About Us Without Us": Reading Protests against Oppressive Knowledge Production as Guidelines for Solidarity Research, Journal of Contemporary Ethnography, 49, 1, pp. 58-85.

YOUNG, I. M. (2005): On female body experience: "Throwing like a girl" and other essays, Oxford, Oxford University Press. 


\section{ANEXO}

\begin{tabular}{|c|c|c|}
\hline \multicolumn{2}{|c|}{ FICHA DEL DISEÑ DE LA INVESTIGACIÓN FEMINISTA } \\
\hline ¿Quién investiga? & ¿Qué se investiga? & ¿Para qué? \\
\hline & & \\
\hline ¿Con quién/es? & \\
\hline ¿Cómo? & Formas de reflexividad \\
\hline Procesos de investigación visibles & \\
\hline & & \\
\hline & \\
\hline Procesos de investigación invisibles & \\
\hline & \\
& \\
\hline
\end{tabular}

Ficha de diseño de la investigación feminista 